\title{
Mastering RNAi in mice
}

In vivo RNAi is a powerful tool for studying gene function, but its application in mice has been limited by the unreliability of methods for making the necessary transgenic animals. These problems have been overcome in a recent paper that reports an economical and efficient approach to generating mice that express short hairpin RNAs (shRNAs), which trigger RNAi. The authors describe how the conditional and reversible nature of the knockdown provides new avenues for using mice to model disease.

Until now, the production of transgenic mice expressing shRNAs has relied on pronuclear DNA injection or viral transduction, but the knockdown achieved is highly variable owing to the unpredictable nature of the transgene insertion. An alternative is to use homologous recombination to make transgenic mice, but this is an inefficient process.

Premsrirut, Dow and colleagues aimed to develop a system that would overcome these limitations and provide the ability to both monitor and regulate gene knockdown. They used an expression cassette that contains several elements: a microRNA backbone into which any shRNA sequence can be introduced, a GFP reporter and target sites for the Flp/FRT recombinase system. The shRNA expression construct is introduced into embryonic stem cells (ESCs) that carry a 'homing' cassette, into which the shRNA transgene is targeted by site-specific Flp/FRT recombination.

\section{By making in vivo RNAi} in mice efficient, scalable, inducible and reversible, this paper provides an important new tool for mouse functional genetics

The homing cassette puts the shRNA transgene under the control of a doxycycline (DOX)-inducible promoter. It also includes a selectable marker that is expressed only if a single copy of the shRNA expression cassette is correctly inserted. This approach allowed the authors to generate ESCs containing correctly targeted expression cassettes rapidly and efficiently for a large number of shRNAs.

Gene knockdown was then tested in a range of these transgenic ESCs, in mice produced from these cells and in cell lines derived from these mice. Knockdown was potent following addition of DOX, could be monitored by GFP expression and was reversible on removal of DOX. Furthermore, the knockdown phenotypes mimicked those resulting from deletions of the target genes. With a slight modification of the initial system, robust shRNA expression was achieved in almost all adult mouse tissues.
The authors went on to show how the inducible and reversible nature of the knockdown system can be used to gain new insights into gene function in development and disease. For example, they used it to knock down adenomatous polyposis coli (APC) - a key component of WNT signalling and a tumour suppressor - for specific intervals during development, revealing new insights into its function that could not be gained using conditional deletions. They also used a restricted period of APC knockdown to generate mice with lymphomas and showed that the cancerous phenotype was partially reversed following DOX withdrawal. Such tests of the reversibility of disease phenotypes could prove useful in testing potential therapies.

By making in vivo RNAi in mice efficient, scalable, inducible and reversible, this paper provides an important new tool for mouse functional genetics. As the authors point out, the ability to do more complex studies, in which several transgenes are introduced into the same animal, is an important goal for more realistic disease modelling. Towards this goal, they point to - and show proof of principle for - efficient strategies for generating such animals, based on rederiving ESCs from transgenic animals and introducing further transgenes into these cells. This approach avoids the need for more time-consuming methods involving multiple crosses and should speed up the development of new mouse models.

Louisa Flintoft

ORIGINAL RESEARCH PAPER Premsrirut, P. K. et al. A rapid and scalable system for studying gene function in mice using conditional RNA interference. Cell 145, 145-158 (2011) 